\title{
Peripheral and central mechanisms of chronic musculoskeletal pain
}

\author{
Kathleen A Sluka* \\ Kathleen A Sluka: kathleen-sluka@uiowa.edu \\ *Department of Physical Therapy \& Rehabilitation Science, 1-248 MEB, University of lowa, lowa \\ City, IA 52242, USA
}

\section{How helpful have animal models been in investigations into the peripheral \& central mechanisms of chronic musculoskeletal pain?}

\begin{abstract}
Animal models have been extremely helpful in gaining a better understanding of both peripheral and central mechanisms in chronic musculoskeletal pain. There are a number of animal models of musculoskeletal pain that have been developed to mimic different pain conditions. These models include those of muscle or joint inflammation (acute and chronic), noninflammatory centrally maintained pain, overuse syndrome and exercise-induced pain [1-5]. By matching the appropriate model with the clinical pain condition, scientists have begun to decipher both peripheral and central mechanisms of chronic musculoskeletal pain. As an example, recent work from our laboratory shows a significant role in acid-sensing ion channels in the peripheral nociceptors innervating muscles and joints in maintaining inflammatory pain, but not in maintaining central pain [6,7]. On the other hand, we have shown that in the noninflammatory model (induced by repeated acid injections) once hyperalgesia has developed it is independent of nociceptive input, and is maintained by sensitization of neurons in the CNS [8]. Understanding the mechanisms in both the peripheral nervous system and CNS, and the differences between disease models, should help to develop better treatments for improved management of chronic musculoskeletal pain and to prevent the transition from acute to chronic pain.
\end{abstract}

\section{How prevalent is this type of pain in the population \& what are the current treatment options available to patients?}

\begin{abstract}
Almost everyone will experience pain of the muscles or joints at some point in their lives. Chronic musculoskeletal pain affects between 20 and $50 \%$ of the population; in general, women experience more chronic musculoskeletal pain than men. The CDC report the incidence of different types of chronic musculoskeletal pain as being relatively high, with 28, 15 and 30\% experiencing back, neck and joint pain, respectively [101]. Despite its prevalence, treatments for chronic musculoskeletal pain are aimed at managing the pain rather than curing the pain. Pharmacologically, treatment with anti-inflammatories, antidepressants and anticonvulsants are often used to control different aspects of the pain. Nonpharmacologically, both psychological and physical management techniques are
\end{abstract}

\footnotetext{
(C) 2013 Future Medicine Ltd

Financial \& competing interests disclosure: The author has no relevant affiliations or financial involvement with any organization or entity with a financial interest in or financial conflict with the subject matter or materials discussed in the manuscript. This includes employment, consultancies, honoraria, stock ownership or options, expert testimony, grants or patents received or pending, or royalties.

No writing assistance was utilized in the production of this manuscript.
} 
effective. One of the most effective treatments for chronic musculoskeletal pain is regular exercise, this can be in the form of walking, cycling, swimming or just by increasing physical activity levels [9]. Despite its effectiveness, compliance with a regular exercise program is poor and exercise can initially increase pain in people with chronic pain [10] Future research should be geared toward techniques to improve compliance and treatments to reduce exercise-induced pain.

\section{What are the techniques available to scientists for the study of peripheral \& central mechanisms of chronic musculoskeletal pain?}

Over the last several decades, the techniques available to study mechanisms of pain in general have grown exponentially. Techniques in neurobiology are readily employed to study animal models of pain, as well as pain in human subjects. In general, these techniques are used to examine both peripheral and central changes.

In animals, commonly used outcome measures include behavioral techniques aimed at understanding simple and complex pain behaviors, or directly recording neuronal activity of the nociceptors, its cell body in the dorsal root ganglia or central neurons. These behavioral and electrophysiological techniques are often used by scientists in combination with pharmacological and genetic techniques to decipher potential new targets for treatment of chronic muscle pain. To examine potential neurotransmitters and receptors, or intracellular pathways involved in the transmission of painful information, scientists have used pharmacological blockade or activation, and genetic modification of the target.

Pharmacological blockade allows scientists to test particular pathways at the site of insult, or at any site along the central pain pathways. Using pharmacology, the time course of involvement of a particular target is examined by blocking prior to the development of hyperalgesia, or at some time period after the development of hyperalgesia. Using genetic techniques, such as knockout mice, scientists can examine the role of a particular target that does not have specific pharmacological agents to activate or block the gene. Knockout mice remove the gene from the whole body and, thus, the site of activation is not usually known. However, development of conditional knockouts or viral-vector delivery of siRNA can knockdown genes in particular sites or cell types. Together, these techniques provide strong evidence for proposed neurotransmitters, receptors or intracellular pathways to allow scientists to identify new potential pharmacological targets. These techniques also allow scientists to examine mechanisms of action of existing treatment, such as exercise, to develop better treatment protocols for improved effectiveness.

Confirmation of changes in the target gene or protein as a result of injury is often achieved using real-time polymerase chain reaction (mRNA), western Blot analysis (protein) or immunohistochemistry (protein). Scientists can directly record from isolated dorsal root ganglia neurons or central neurons along the pain pathway to directly examine changes in the neuron activity or specific receptors. Activity can also be measured by examining for changes in intracellular ion concentrations, such as calcium. Using the same pharmacological and genetic manipulations, the specific target gene or protein can be analyzed for its role in modifying neuronal activity. Thus, in animal models of musculoskeletal pain, a variety of available techniques can be employed to better understand peripheral and central mechanisms underlying pain.

In human subjects, a number of parallel psychophysical measures are used to translate animal data to human subjects. In addition, human subjects can be directly asked to evaluate their pain under a variety of situations. Measurement of resting pain, pain with movement, pain thresholds, pain tolerance and pain ratings to a fixed noxious stimulus are often used. Temporal summation, an increased pain rating to a repetitive fxed noxious stimulus, 
measures the excitability of the CNS and is increased in patients with chronic musculoskeletal pain. Conditioned pain modulation, analgesia to a noxious stimuli, is used to measure the inhibitory capability of the CNS and is decreased in individuals with chronic musculoskeletal pain. Using experimental pain models in human subjects, which would control the noxious input, the variability of pain and the underlying constructs that contribute to pain variability can be examined. Experimental pain models have proven useful for understanding genetic and psychological constructs that underlie the development of musculoskeletal pain. Applying a battery of psychophysical tests in healthy human subjects and those with chronic pain has allowed scientists to gain a better understanding of the constructs involved in the development of chronic pain, as well as the continuation of chronic pain.

\section{From studies conducted so far, could you briefly describe how peripheral \& central mechanisms of chronic musculoskeletal pain differ \& whether there is any overlap between these two mechanisms?}

Chronic musculoskeletal pain clearly involves activation of both peripheral and central pain pathways. In some patients the pain is primarily maintained by peripheral inputs while in others the pain is maintained primarily by central changes; however, the majority of patients probably have a combination of both peripheral and central changes. Furthermore, changes in the CNS can drive alterations in the peripheral nervous system, and vice versa. Thus, deciphering the mechanisms in an individual can be incredibly difficult. It has become increasingly clear that pain outside the site of injury, referred pain and secondary hyperalgesia, reflects alterations in the CNS; however, in some cases symptoms are driven by continued input from nociceptors. On the other hand, pain at the site of injury generally reflects changes in the peripheral nervous system; however, in some cases these symptoms are enhanced by alterations in the CNS.

For chronic musculoskeletal pain, the mechanisms underlying the conditions are dependent on the disease or syndrome - osteoarthritis is different from fibromyalgia, which is different from rheumatoid arthritis. Thus, the involvement of peripheral and central pain pathways can be unique to the pain condition. For example, inflammatory joint pain, as observed in rheumatoid arthritis, has a strong peripheral component that involves sensitization of nociceptors by inflammatory cytokines present as a result of the inflammation (e.g., interleukins and TNF). On the other hand, noninflammatory muscle pain does not show local inflammation, but rather has a strong central pain component that is independent of peripheral afferent input $[5,8]$. In patients with osteoarthritis, the majority have a peripheral nociceptor-driven pain that is eliminated when the joint is replaced. However, approximately $20 \%$ of subjects have a strong central component to their pain, with enhanced temporal summation and loss of central inhibition that remains after joint replacement $(>1$ year) $[11,12]$. Understanding the differences in underlying mechanisms between those who fully recover to a pain-free joint after surgery and those with significant pain and disability will allow us to tailor treatments to individuals with the same condition to improve outcome.

Individual variability in pain processing results in significant variability in the response to a given stimuli, such that some people will report very little pain while others report maximal pain under the same conditions. This individual variability is a result of many factors, including genetic, psychological, biologic, sex, age, environmental and sociocultural factors [13]. All of these factors can directly influence how a nociceptor or a central neuron responds to a noxious stimuli or disease process. Regardless of the underlying disease process, these factors significantly influence pain and the outcome of treatment. Some of these factors are modifiable (e.g., psychologic, biologic, environmental and sociocultural) 
while others are not (e.g., genetic, sex and age). Therefore, modifiable factors provide an additional potential target for designing individualized treatment strategies. Future research should focus on the factors that lead to individual variability in pain processing and how tailoring treatments to these modifiable factors will improve pain and quality of life.

\section{What has your research highlighted regarding the role that muscle fatigue plays in chronic musculoskeletal pain?}

Physical and mental fatigue are common comorbid symptoms in chronic pain patients, particularly those with chronic musculoskeletal pain. This fatigue can significantly impact an individual's physical activity levels and their quality of life. Therefore, understanding the role of physical fatigue and its interactions with pain is critical to better treatment. We have developed animal models of whole body physical fatigue that, when given in combination with a subthreshold muscle insult, results in long-lasting widespread pain [14-16]. Our research is beginning to examine the underlying mechanisms of how fatigue and pain interact. We show that both fatigue and muscle insult activate neurons in the brainstem more specifically. Our data further show that blocking glutamate receptors in the brainstem during the fatigue task prevents hyperalgesia in this fatigue-induced pain model. Interestingly, hyperalgesia that occurs with muscle fatigue combined with muscle insult only presents in sedentary animals and does not occur if animals are physically active. The activation of neurons in the brainstem by the fatigue task does not occur in physically active animals. To translate the animal findings, we recently tested the effects of the physical fatigue task in individuals with fibromyalgia and compared with healthy controls [17]. There is significantly increased pain and physical fatigue during the task in individuals with fibromyalgia; however, both healthy controls and fibromyalgia subjects perform equally well on the fatigue task. Together, these data point to a common central pathway and mechanism mediating the pain and fatigue associated with chronic musculoskeletal pain.

\section{How would you like to see this area of study within pain management progress in the future?}

Scientists need to be aware of the clinical conditions and the clinical manifestation of chronic pain in order to translate these into animal models of pain. For example, people with chronic musculoskeletal pain have significant pain during movement, which limits physical activity levels and participation in effective exercise programs. Some conditions, such as osteoarthritis, may have relatively low levels of resting or spontaneous pain. Development of behavioral assays that mimic this decreased physical activity and exercise-induced pain would help in developing treatments for chronic musculoskeletal pain. For further translation, clinical trials should also consider the appropriate measures that are specific to the pain condition being studied. Some treatments may be more effective for different types of pain (resting vs movement), or for different manifestations of the disease (pain vs fatigue). Recently, we showed that transcutaneous electrical nerve stimulation, a nonpharmacological treatment for pain, reduced pain during movement but not at rest in people with fibromyalgia, and reduced hyperalgesia but not resting pain in people with osteoarthritis $[18,19]$. We also demonstrated that after a total knee replacement, predictors of postoperative movement pain were different from those for postoperative resting pain [20]. Thus, understanding the differences between different manifestations of chronic musculoskeletal pain and different pain syndromes will allow us to tailor current therapies and develop disease-specific therapeutics. 


\section{References}

1. Barr AE, Barbe MF. Pathophysiological tissue changes associated with repetitive movement: a review of the evidence. Phys Ther. 2002; 82:173-187. [PubMed: 11856068]

2. Christianson CA, Corr M, Mobargha A, Firestein GS, Yaksh TL, Svensson CI. Characterization of the acute and persistent pain state present in $\mathrm{K} / \mathrm{B} \times \mathrm{N}$ serum transfer arthritis: persistent neuropathic pain triggered by inflammatory arthritis. Pain. 2010; 151(2):394-403. [PubMed: 20739123]

3. Radhakrishnan R, Moore SA, Sluka KA. Unilateral carrageenan injection into muscle or joint induces chronic bilateral hyperalgesia in rats. Pain. 2003; 104:567-577. [PubMed: 12927629]

4. Schaible HG, Grubb BD. Afferent and spinal mechanisms of joint pain. Pain. 1993; 55:5-54. [PubMed: 8278210]

5. Sluka KA, Kalra A, Moore SA. Unilateral intramuscular injections of acidic saline produce a bilateral, long-lasting hyperalgesia. Muscle Nerve. 2001; 24:37-46. [PubMed: 11150964]

6. Gautam M, Benson CJ, Ranier JD, Light AR, Sluka KA. ASICs do not play a role in maintaining hyperalgesia induced by repeated intramuscular acid injections. Pain Res Treat. 2012; 2012:817347. [PubMed: 22191025]

7. Walder RY, Rasmussen LA, Rainier JD, Light AR, Wemmie JA, Sluka KA. ASIC1 and ASIC3 play different roles in the development of hyperalgesia after inflammatory muscle injury. J Pain. 2010; 11:210-218. [PubMed: 20015700]

8. Da Silva LF, Desantana JM, Sluka KA. Activation of NMDA receptors in the brainstem, rostral ventromedial medulla, and nucleus reticularis gigantocellularis mediates mechanical hyperalgesia produced by repeated intramuscular injections of acidic saline in rats. Pain. 2010; 11:378-387.

9. Bement, MK. Exercise-induced hypoalgesia: an evidence-based review. In: Sluka, KA., editor. Mechanisms and Management of Pain for the Physical Therapist. IASP Press; Seattle, WA, USA: 2009. p. 143-166.

10. Staud R, Robinson ME, Price DD. Isometric exercise has opposite effects on central pain mechanisms in fibromyalgia patients compared to normal controls. Pain. 2005; 118:176-184. [PubMed: 16154700]

11. Arendt-Nielsen L, Nie H, Laursen MB, et al. Sensitization in patients with painful knee osteoarthritis. Pain. 2010; 149:573-581. [PubMed: 20418016]

12. O'Connor MI. Implant survival, knee function, and pain relief after TKA. Are there differences between men and women? Clin Orthop Relat Res. 2011; 469(7):1846-1851. [PubMed: 21267799]

13. Frey Law, LA.; George, SZ. Individual differences and pain variability. In: Sluka, KA., editor. Pain Mechanisms and Managment for the Physical Therapist. IASP Press; Seattle, WA, USA: 2009. p. 73-94.

14. Sluka KA, Danielson J, Rasmussen L, Dasilva LF. Exercise-induced pain requires NMDA receptor activation in the medullary raphe nuclei. Med Sci Sports Exerc. 2012; 44:420-427. [PubMed: 21795998]

15. Sluka KA, Rasmussen LA. Fatiguing exercise enhances hyperalgesia to muscle inflammation. Pain. 2010; 148:188-197. [PubMed: 19632780]

16. Yokoyama T, Lisi TL, Moore SA, Sluka KA. Muscle fatigue increases the probability of developing hyperalgesia in mice. J Pain. 2007; 8:692-699. [PubMed: 17627895]

17. Dailey, DL.; Sluka, KA.; Rakel, BA.; Keffala, VJ. Pain, cognitive fatigue, and physical fatigue in individuals with fibromyalgia and healthy controls. Presented at: 14th World Congress on Pain; Milan, Italy. 27-31 August 2012;

18. Dailey DL, Vance CGT, Liebano RE, et al. Transcutaneous Electrical Nerve Stimulation (TENS) reduces and improves function in people with fibromyalgia. J Pain. 2012; 12(4 Suppl):23.

19. Vance CG, Rakel BA, Blodgett NP, et al. Effects of transcutaneous electrical nerve stimulation on pain, pain sensitivity, and function in people with knee osteoarthritis: a randomized controlled trial. Phys Ther. 2012; 92(7):898-910. [PubMed: 22466027]

20. Rakel BA, Blodgett NP, Bridget ZM, et al. Predictors of postoperative movement and resting pain following total knee replacement. Pain. 2012; 153:2192-2203. [PubMed: 22840570] 


\section{Website}

101. CDC. National Center for Health Statistics, Health, United States, 2007 with Chartbooks on Trends in the Health of American. www.cdc.gov/nchs/data/hus/hus07.pdf

\section{Biography}

Kathleen A Sluka: Dr Sluka is a professor in the Department of Physical Therapy and Rehabilitation Science at the University of Iowa (IA, USA). She is also a member of the Pain Research Program and the Neuroscience Graduate Program. She received a physical therapy degree from Georgia State University (GA, USA) and practiced physical therapy pain management in Houston (TX, USA) before obtaining a PhD in Anatomy from the University of Texas Medical Branch in Galveston (TX, USA). After a postdoctoral fellowship with Dr William D Willis, she joined the faculty at the University of Iowa. Dr Sluka's research focuses on the neurobiology of musculoskeletal pain, as well as the mechanisms and effectiveness of nonpharmacological pain treatments commonly used by physical therapists. She has published over 140 peer-reviewed manuscripts, numerous book chapters and a textbook on Pain Mechanisms and Management for the Physical Therapist. She has received numerous awards including the Marian Williams Award for Research in Physical Therapy and is a Catherine Worthingham Fellow from the American Physical Therapy Association and the Frederick WL Kerr Basic Science Research Award from the American Pain Society. She is actively involved in the International Association for the Study of Pain, the American Pain Society and the American Physical Therapy Association, serving on committees, task forces and society boards.

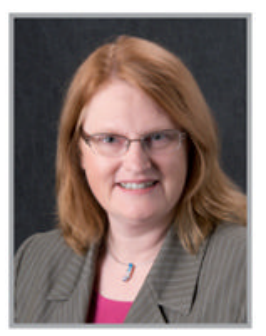

